\title{
Technique: open lumbar decompression and fusion with the Excelsius GPS robot
}

\author{
Corinna C. Zygourakis, MD, ${ }^{1}$ A. Karim Ahmed, BS, ${ }^{1}$ Samuel Kalb, MD, ${ }^{1,2}$ Alex M. Zhu, PA-C, ${ }^{1}$ \\ Ali Bydon, MD, ${ }^{1}$ Neil R. Crawford, PhD, ${ }^{2}$ and Nicholas Theodore, MD ${ }^{1}$ \\ 1Department of Neurosurgery, Johns Hopkins School of Medicine, Baltimore, Maryland; and 2Division of Neurological Surgery, \\ Barrow Neurological Institute, St. Joseph's Hospital and Medical Center, Phoenix, Arizona \\ The Excelsius GPS (Globus Medical, Inc.) was approved by the FDA in 2017. This novel robot allows for real-time intra- \\ operative imaging, registration, and direct screw insertion through a rigid external arm-without the need for interspinous \\ clamps or K-wires. The authors present one of the first operative cases utilizing the Excelsius GPS robotic system in \\ spinal surgery. A 75-year-old man presented with severe lower back pain and left leg radiculopathy. He had previously \\ undergone 3 decompressive surgeries from $L 3$ to $L 5$, with evidence of instability and loss of sagittal balance. Robotic \\ assistance was utilized to perform a revision decompression with instrumented fusion from $L 3$ to $S 1$. The usage of \\ robotic assistance in spinal surgery may be an invaluable resource in minimally invasive cases, minimizing the need for \\ fluoroscopy, or in those with abnormal anatomical landmarks.
}

The video can be found here: https://youtu.be/yVI-sJWf9lw.

KEYWORDS robotic spine surgery; Excelsius GPS; lumbar decompression; video 